Sudden transition from equilibrium stability to chaotic dynamics in a cautious tâtonnement model

This content has been downloaded from IOPscience. Please scroll down to see the full text.

2016 J. Phys.: Conf. Ser. 692012005

(http://iopscience.iop.org/1742-6596/692/1/012005)

View the table of contents for this issue, or go to the journal homepage for more

Download details:

IP Address: 79.17.96.135

This content was downloaded on 22/01/2017 at 08:44

Please note that terms and conditions apply.

You may also be interested in:

Chaotic Dynamics of a Periodically Modulated JosephsonJunction

Wu Qin and Li Fei

Analytical and Numerical Studies for ChaoticDynamics of a Duffing Oscillator with a Parametric

Force

Wang Yan-Qun and Wu Qin

Correlation between Chaotic Dynamics and Level Spacings: theLyapunov and Dixon Dip Approaches to Highly Excited Vibration ofDeuterium Cyanide

Ou Shu-Ching and Wu Guo-Zhen

Effects of Atomic Coherence and Injected ClassicalField on Chaotic Dynamics of Non-degenerate

Cascade Two-PhotonLasers

Zhang Ya-Jun, Huang Hong-Bin, Yang Peng et al.

Dynamical Systems in Cosmology

M Szydlowski

Accessibility to second stability regime of high $\mathrm{n}$ ballooning modes

H. Capes, C. Mercier and J.P. Morera

A memristor oscillator based on a twin-T network

Li Zhi-Jun and Zeng Yi-Cheng

Chaotic Dynamics of a Josephson Junction with Nonlinear Damping

Li Fei, Pan Chang-Ning, Zhang Dong-Xia et al. 


\title{
Sudden transition from equilibrium stability to chaotic dynamics in a cautious tâtonnement model
}

\author{
I. Foroni ${ }^{1}$, A. Avellone ${ }^{1}$ and A. Panchuk ${ }^{2}$ \\ E-mail: ilaria.foroni@unimib.it, alessandro.avellone@unimib.it, \\ anastasiia.panchuk@gmail.com \\ ${ }^{1}$ Department of Statistica e Metodi Quantitativi University of Milano-Bicocca (Italy) \\ ${ }^{2}$ Institute of Mathematics, NAS of Ukraine, 3 Tereshchenkivska str., 01601 Kyiv (Ukraine)
}

\begin{abstract}
Discrete time price adjustment processes may fail to converge and may exhibit periodic or even chaotic behavior. To avoid large price changes, a version of the discrete time tâtonnement process for reaching an equilibrium in a pure exchange economy based on a cautious updating of the prices has been proposed two decades ago. This modification leads to a one dimensional bimodal piecewise smooth map, for which we show analytically that degenerate bifurcations and border collision bifurcations play a fundamental role for the asymptotic behavior of the model.
\end{abstract}

\section{Introduction}

In [12] the author observed that while habitually the eonomic models used to describe the discrete time tâtonnement process allowed very large price changes, "in reality price changes are usually restricted in a given period and are adjusted to substantial market disequilibria only over time" (p.1 in [12]). To overcome the shortcoming he suggested the assumption of a cautious process of price adjustment, that is, he proposed introducing a bound on the percentage price changes in function of the excess of the demand. As an example, he inserted the cautious mechanism in the simplest case of pure exchange economy introduced in the work of Day and Pianigiani $([2])$. The analytical description of the model resulted expressed by a bimodal 1D continuous piecewise linear map which showed the possibility of erratic dynamics even in presence of the cautious adjustment. In the following years, the model has been extensively studied by many authors in consideration of its economic implications. Nonetheless, from the mathematical point of view the map describing the original model has not yet been completely explained, mainly because of the fact that the map is not smooth. To our knowledge, the bifurcation structure of the parameter space of a generic 1D piecewise linear bimodal map has been described to a large extent $[9,10]$. Unlike the skew tent map, the piecewise linear bimodal map has two border points, which implies more tangled bifurcation structure of its parameter space. However, for certain parameter values the bimodal map (or its suitable iterate) admits reduction either to a skew tent map or to a discontinuous map defined in two partitions. The results stated in these two references are exploited in the present paper in order to describe asymptotic behavior of the map which models a cautious tâtonnement process. This paper is an abridged version of [3] to which we would like to refer interested readers for analytical proofs and details. 


\section{The model}

The model framework considers a pure exchange economy with 2 individuals, which we label with an index $i(i=1,2)$, and 2 commodities. The commodities have to be chosen in amounts $y$ and $w$, respectively, and we assume that individuals' preferences are characterized by the following Cobb-Douglas utility functions

$$
\begin{aligned}
& U^{1}(y, w)=y^{\alpha} w^{1-\alpha}, \\
& U^{2}(y, w)=y^{\beta} w^{1-\beta}
\end{aligned}
$$

where $0<\alpha, \beta<1$. Moreover, we consider that individual 1 is endowed with a quantity $A$ of the first good while individual 2 is endowed with a quantity $B$ of the second good. We indicate with $p$ the unitary price of the first good while $q$ denote the unitary price of the second good. Thus, we may express the budget constraint of consumer 1 as

$$
p y+q w \leq p A
$$

and the budget constraint of consumer 2 as

$$
p y+q w \leq q B .
$$

Let us suppose that prices are normalized so that the price $q$ of the second good is set to a constant and only the price $p$ of the first is adjusted, then maximizing (1) subject to (3) and (2) subject to (4) we obtain the following total excess of demand for the first good

$$
z(p)=-(1-\alpha) A+\frac{\beta q B}{p} .
$$

To introduce the tâtonnement process, we have to substitute $p_{t}$ for $p$ in (5) and then consider the difference equation

$$
p_{t+1}=\max \left\{0, g\left(z\left(p_{t}\right)\right)\right\},
$$

where $g\left(z\left(p_{t}\right)\right)$ expresses the price adjustment process as a function of the excess of demand $z\left(p_{t}\right)$. In particular, choosing the relative price adjustment mechanism

$$
g\left(z\left(p_{t}\right)\right)=p_{t}\left(1+\lambda z\left(p_{t}\right)\right),
$$

where $\lambda$ is a positive constant representing the velocity of adjustment, expression (6) becomes

$$
p_{t+1}=\max \left\{0, p_{t}\left(1+\lambda z\left(p_{t}\right)\right)\right\} .
$$

To obtain exactly the model proposed in [12], we have to assume the hypothesis that prices are adjusted cautiously meaning that the amount of the percentage price changes is supposed to be bounded. Analytically this assumption can be expressed adding the restrictions

$$
1-r \leq \frac{g\left(z\left(p_{t}\right)\right)}{p_{t}} \leq 1+r
$$

where $0<r<1$ denotes the maximal rate of price change. Thus, by substituting (9) in (6) and after some manipulations, the price adjustment mechanism describing the evolution of the model is defined by the following difference equation

$$
p_{t+1}=\max \left\{(1-r) p_{t}, \min \left\{(1+r) p_{t},\left(\lambda \beta q B+(1-\lambda(1-\alpha) A) p_{t}\right)\right\}\right\} .
$$


By assuming the condition $\beta=1-\alpha$ as in [12] we can rewrite (10) as a $1 \mathrm{D}$ continuous piecewise linear map, $p_{t+1}=g\left(p_{t}\right)$, with $g:[0, \infty) \rightarrow[0, \infty)$ defined by three linear functions $g_{\mathcal{L}}, g_{\mathcal{M}}$, and $g_{\mathcal{R}}$ as follows

$$
g: p \mapsto g(p)=\left\{\begin{array}{clc}
g_{\mathcal{L}}(p)=(1+r) p & \text { for } & 0 \leq p \leq \frac{\varepsilon \gamma}{A(r+\varepsilon)}, \\
g_{\mathcal{M}}(p)=\frac{\varepsilon \gamma}{A}+(1-\varepsilon) p & \text { for } & \frac{\varepsilon \gamma}{A(r+\varepsilon)}<p \leq \frac{\varepsilon \gamma}{A(\varepsilon-r)}, \\
g_{\mathcal{R}}(p)=(1-r) p & \text { for } & p>\frac{\varepsilon \gamma}{A(\varepsilon-r)},
\end{array}\right.
$$

where $r, \varepsilon, A$, and $\gamma$ are real parameters such that

$$
0<r<1, \quad \varepsilon \equiv \lambda A \beta>r, \quad A>0, \quad \gamma=q B>0 .
$$

It can be proved (see [3] for details) that the map $g$ is topologically conjugate to the following reduced map

$$
f: x \rightarrow f(x)= \begin{cases}f_{\mathcal{L}}(x)=a_{\mathcal{L}} x+\mu_{\mathcal{L}}=(1+r) x, & 0 \leq x \leq x_{\mathcal{L}} \\ f_{\mathcal{M}}(x)=a_{\mathcal{M}} x+\mu_{\mathcal{M}}=(1-\varepsilon) x+\varepsilon, & x_{\mathcal{L}}<x \leq x_{\mathcal{R}} \\ f_{\mathcal{R}}(x)=a_{\mathcal{R}} x+\mu_{\mathcal{R}}=(1-r) x, & x>x_{\mathcal{R}}\end{cases}
$$

where

$$
a_{\mathcal{L}}=1+r, \quad a_{\mathcal{M}}=1-\varepsilon, \quad a_{\mathcal{R}}=1-r \quad \text { and } \quad \mu_{\mathcal{L}}=\mu_{\mathcal{R}}=0, \quad \mu_{\mathcal{M}}=\varepsilon
$$

are the slopes and the offsets, respectively, while

$$
x_{\mathcal{L}}=\frac{\varepsilon}{\varepsilon+r}, \quad x_{\mathcal{R}}=\frac{\varepsilon}{\varepsilon-r}
$$

are the border points. Therefore, asymptotic dynamics of $f$ is qualitatively the same as asymptotic dynamics of $g$, while the factor $\frac{\gamma}{A}$ only influences the scale. As a consequence, below we restrict our analysis to the $(r, \varepsilon)$ parameter plane. The map $f$ in (13) is bimodal as the branches $f_{\mathcal{L}}$ and $f_{\mathcal{R}}$ are monotonically increasing while the middle one, $f_{\mathcal{M}}$, is monotonically decreasing. By definition the border points $x_{\mathcal{L}}$ and $x_{\mathcal{R}}$ are both singular points for $f$. Moreover, $f$ has a local maximum in $x_{\mathcal{L}}$, and a local minimum in $x_{\mathcal{R}}$, which are denoted as

$$
x_{M} \stackrel{d f}{=} f\left(x_{\mathcal{L}}\right)=\frac{\varepsilon(r+1)}{\varepsilon+r} \quad x_{m} \stackrel{d f}{=} f\left(x_{\mathcal{R}}\right)=\frac{\varepsilon(1-r)}{\varepsilon-r} .
$$

\section{Bifurcation structures in the parameter space}

In this section, we begin the analysis of the model (10) introducing some of the properties of the map $f$ which describes the price evolution of the first commodity in the economic system. We will show that only two kind of dynamics are allowed since it will be proved that, depending on the values of the parameters, the price or converges to a globally stable equilibrium or follows a chaotic dynamics.

\subsection{Preliminary observations, feasible domain and absorbing intervals}

The region $D$ of definition of the map $f$ is confined by three boundaries:

$$
\delta_{N D}=\{(r, \varepsilon): \varepsilon=r\}, \quad \delta_{r 1}=\{(r, \varepsilon): r=1\}, \quad \delta_{r 0}=\{(r, \varepsilon): r=0\},
$$

that is,

$$
D=\{(r, \varepsilon): \varepsilon>r, 0<r<1\} .
$$


Moreover, the map (13) has a fixed point $x^{0}$ in the origin which is always repelling. Due to this reason we restrict our analysis to the interval $(0, \infty)$ which is divided into the three partitions, $I_{\mathcal{L}}=\left(0, x_{\mathcal{L}}\right], I_{\mathcal{M}}=\left(x_{\mathcal{L}}, x_{\mathcal{R}}\right]$, and $I_{\mathcal{R}}=\left(x_{\mathcal{R}}, \infty\right)$, on which the functions $f_{\mathcal{L}}, f_{\mathcal{M}}$, and $f_{\mathcal{R}}$ are defined. For parameter values belonging to the feasible domain $D$ given in (17) the map $f$ always admits an absorbing interval $J$ which is globally attracting. The existence of such an interval is a very important feature for the system, because all orbits are trapped by the absorbing set, and what happens in this set determines the long-run dynamics of the model. These preliminary results prove an important economic property of the model since we may affirm that for the values of the parameters considered it is not possible that price $p$ becomes zero or diverges. Moreover, the interval in which the price varies may exclude big jumps if the relative price adjustment is restricted by a maximal rate of increase or decrease. The investigation of the absorbing interval of $f$ allows us to identify which ones of the branches defined in (13) are involved in the asymptotic behavior of $f$. Thus, $D$ may be divided into three sub-regions denoted by $D_{\mathcal{M}}, D_{\mathcal{L M}}$, and $D_{\mathcal{L M R} \text {. These regions distinguish situations in which different numbers of branches of } f \text { are }}$ involved in its asymptotic dynamics as reflected by the subscripts. That is, asymptotic behavior of the map $f$ involves only the middle branch $\left(D_{\mathcal{M}}\right)$, the left and the middle branches $\left(D_{\mathcal{L M}}\right)$, or all three branches $\left(D_{\mathcal{L M R}}\right)$. According to this, we denote the absorbing interval of $f$ by $J_{\mathcal{M}}$, $J_{\mathcal{L M}}$, or $J_{\mathcal{L M R}}$ when we consider parameter values in $D_{\mathcal{M}}, D_{\mathcal{L M}}$ or $D_{\mathcal{L M R}}$, respectively.

\subsection{A stable fixed point: region $D_{\mathcal{M}}$}

Apart from the repelling origin $x^{0}$, the map $f$ always has another fixed point $x^{*}=1$ which may be stable or unstable. By definition $x^{*}$ is stable when the related slope $\left|f^{\prime}\left(x^{*}\right)\right|=\left|a_{\mathcal{M}}\right|<1$, which clearly holds whenever $\varepsilon<2$. The line

$$
\delta_{D F B}=\left\{(r, \varepsilon): \varepsilon=\varepsilon_{D F B}=2\right\}
$$

defines a degenerate flip bifurcation (DFB) [11] boundary in the parameter plane of $f$. Thus, the point $x^{*}$ is stable for the parameter values belonging to region $D_{\mathcal{M}}=\{(r, \varepsilon): 0<r<1, r<\varepsilon<$ $2\}$ confined by three feasible domain boundaries $\delta_{N D}, \delta_{r 0}, \delta_{r 1}$, and the DFB boundary $\delta_{D F B}$. Moreover, since the slope of the left branch is $a_{\mathcal{L}}>1$ and the slope of the right branch is $a_{\mathcal{R}}<1$, any orbit of $f$ enters the middle partition $I_{\mathcal{M}}$ in a finite number of iterations, and hence, the point $x^{*}$ is globally attracting. Thus, as $\varepsilon \equiv \lambda A \beta$, we can observe that the constant elasticity of substitution of the Cobb-Douglas utility functions, the endowment of the first good and the velocity of the price adjustment mechanism are the parameters influencing the dynamics of the model together with the maximal rate of price change. Furthermore, we can affirm that the values of the parameters $\lambda, A$ and $\beta$ may play a crucial role in preserving the stability of the equilibrium price whatever the value of $r$ is.

\subsection{Reduction to a skew tent map: region $D_{\mathcal{L M}}$}

Immediately after the DFB occurring at $\delta_{D F B}$ the map $f$ has an absorbing interval $J_{\mathcal{L M}}=$ $\left[f^{2}\left(x_{\mathcal{L}}\right), f\left(x_{\mathcal{L}}\right)\right]=J_{\mathcal{L} \mathcal{M}}^{1} \cup J_{\mathcal{L} \mathcal{M}}^{2}$ with

$$
J_{\mathcal{L} \mathcal{M}}^{1}=\left[f^{2}\left(x_{\mathcal{L}}\right), x_{\mathcal{L}}\right]=\left[\frac{\varepsilon(2 r-r \varepsilon+1)}{r+\varepsilon}, \frac{\varepsilon}{r+\varepsilon}\right] \subset I_{\mathcal{L}}
$$

and

$$
J_{\mathcal{L} \mathcal{M}}^{2}=\left(x_{\mathcal{L}}, f\left(x_{\mathcal{L}}\right)\right]=\left(\frac{\varepsilon}{r+\varepsilon}, \frac{\varepsilon(1+r)}{r+\varepsilon}\right] \subset I_{\mathcal{M}} .
$$

Thus, the map $f$ restricted to $J_{\mathcal{L M}}$ is defined as

$$
f_{J_{\mathcal{L M}}}: x \mapsto f_{J_{\mathcal{L M}}}(x)=\left\{\begin{array}{l}
f_{\mathcal{L}}(x)=(1+r) x, \quad \frac{\varepsilon(2 r-r \varepsilon+1)}{r+\varepsilon} \leq x \leq \frac{\varepsilon}{r+\varepsilon} \\
f_{\mathcal{M}}(x)=\varepsilon+(1-\varepsilon) x, \quad \frac{\varepsilon}{r+\varepsilon}<x \leq \frac{\varepsilon(1+r)}{r+\varepsilon}
\end{array}\right.
$$




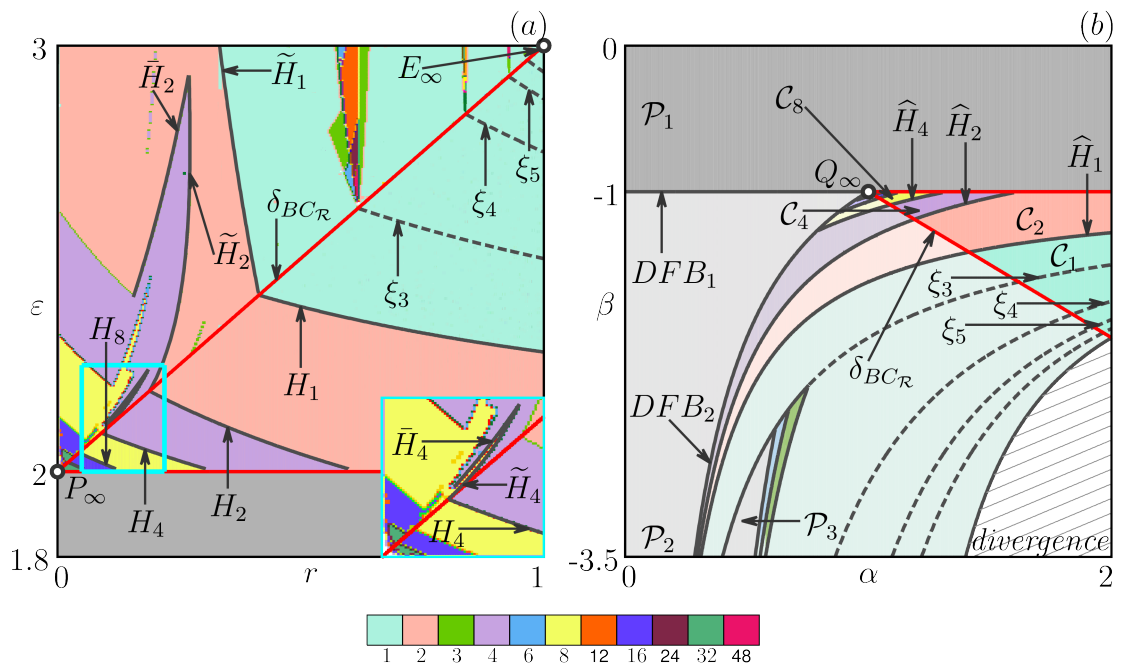

Figure 1. (a) Parameter plane of the skew tent map $\hat{f}$. The region corresponding to $D_{\mathcal{L M}}$ is outlined red. $H_{2^{m}}$ and $\widetilde{H}_{2^{m}}$ are the curves (accumulating at the point $P_{\infty}$ ) where the first homoclinic bifurcation of the repelling cycles $\mathcal{O}_{2^{m}}$ occur. (b) Skew tent structure inside the region $D_{\mathcal{L M}}$ for the map $f$ and its extension to the region $D_{\mathcal{L M R}} . \widehat{H}_{2^{m}}$ indicates the boundaries (accumulating at the point $Q_{\infty}$ ) corresponding to the first homoclinic bifurcation of the repelling cycle $\mathcal{O}_{2^{m}}$. In both panels $\xi_{q}$ designate the fold BCB leading to the appearance of the two unstable cycles $\mathcal{O}_{q}$ and $\widehat{\mathcal{O}}_{q}$.

The map (19) is applicable while the absorbing interval $J_{\mathcal{L M}}$ remains in the left and the middle partitions, that is, until $f\left(x_{\mathcal{L}}\right)=x_{\mathcal{R}}$ is fulfilled and the right endpoint of $J_{\mathcal{L M}}$ coincides with $x_{\mathcal{R}}$. This contact happens when $\varepsilon$ and $r$ belong to the line $\delta_{B C_{\mathcal{R}}}=\{(r, \varepsilon): \varepsilon=r+2\}$, so that $\delta_{B C_{\mathcal{R}}}$ is the boundary between the regions $D_{\mathcal{L M}}$ and $D_{\mathcal{L M R}}$. In such a way the region $D_{\mathcal{L M}}$ is defined as $D_{\mathcal{L M}}=\{(r, \varepsilon): 0<r<1,2<\varepsilon<r+2\}$. The 2D bifurcation diagram shown in Fig. 1(a) displays how the bifurcation structure changes when the parameter point moves from $D_{\mathcal{M}}$ to $D_{\mathcal{L M}}$. In the diagram, gray color indicates convergence of the orbits of the map $f$ to the fixed point $x^{*}$, while the other colors denote regions associated with cyclic chaotic intervals that is chaotic attractors made up of different bands (correspondence between colors and numbers of intervals is indicated in the color bar). As the 2D bifurcation diagram in Fig. 1(a) suggests, the main feature concerning the asymptotic dynamics of (13) in $D_{1} \cup D_{2}$ is that crossing the straight line $\delta_{D F B}(18)$ leads to the abrupt transition from the global stability of the fixed point $x^{*}$ in region $D_{\mathcal{M}}$ to chaos which characterizes the whole area $D_{\mathcal{L M}}$. Thus, the introduction of the cautious price updating in the tâtonnement model causes a sudden change from the stable equilibrium price to the chaotic regime which is rather exceptional economic phenomenon. Not only, but as it will be proved in the following analysis, the complete absence of periodic attracting cycles is a feature of this version of the model that distinguishes it from its classical formulation. As we will show, two characteristics of (13) mainly contribute to the peculiar bifurcation structure of the set $D_{\mathcal{M}} \cup D_{\mathcal{L} \mathcal{M}}$ in the $(r, \varepsilon)$-plane: first, the change in stability of $x^{*}$ when we pass from $D_{\mathcal{M}}$ to $D_{\mathcal{L M}}$, and second, the unimodal shape of $f_{J_{\mathcal{L M}}}$ in (19). The latter fact allows us to assert that the map $f_{J_{\mathcal{L M}}}$ is topologically conjugate to the skew tent map in the normal form

$$
\hat{f}: x \rightarrow \hat{f}(x)= \begin{cases}\hat{f}_{\mathcal{A}}(x)=\alpha x+1, & x \leq 0, \\ \hat{f}_{\mathcal{B}}(x)=\beta x+1, & x>0,\end{cases}
$$


with

$$
\alpha=a_{\mathcal{L}}=1+r, \quad \beta=a_{\mathcal{M}}=1-\varepsilon .
$$

As it is known, the skew tent map has been widely studied by many authors and for its normal form $\hat{f}$ all the possible kinds of border collision bifurcations are classified (see $[4,8,6,7]$ to cite a few, and also [11] for a detailed description and references therein). From the topological conjugacy of (19) and (20) it follows that if we refer to the 2D bifurcation diagram of the skew tent map $\hat{f}$ we may deduce the bifurcation structure of the region $D_{\mathcal{L M}}$ of the map $f$. In particular, we can explain the meaning of the curves that divide $D_{\mathcal{L M}}$ into sub-regions as it is shown in the 2D bifurcation diagram of Fig. 1(a). For that we plot in Fig. 1(b) a bifurcation diagram in the $(\alpha, \beta)$ parameter plane of $\hat{f}$ in order to compare it with the panel (a) (the red line marks the parameter region which corresponds to $\left.D_{\mathcal{L M}}\right)$. As it is immediately seen, the asymptotic dynamics of the skew tent map $\hat{f}$ for the related parameter values corresponds to cyclic chaotic intervals $\mathcal{Q}_{2^{m}}, m=0,1, \ldots$, whose regions are denoted as $\mathcal{C}_{2^{m}}$. It can be also evidenced that after the DFB of the fixed point $x^{*}$ there holds $|\alpha \beta|>1$. Hence, the cycle of period 2 which is born due to the bifurcation is unstable, and therefore the DFB of $x^{*}$ leads to a chaotic regime. For the skew tent map $\hat{f}$ it is known (see, e. g., [11]) that any two contiguous regions $\mathcal{C}_{2^{m}}$ and $\mathcal{C}_{2^{m+1}}$ are separated from each other by the boundary corresponding to the first homoclinic bifurcation of the repelling cycle $\mathcal{O}_{2^{m}}$ of period $2^{m}$. Analytically the homoclinic bifurcation boundary is expressed in general case as

$$
\widehat{H}_{2^{m}}=\left\{(\alpha, \beta): \alpha^{2 \delta_{m}} \beta^{2 \delta_{m+1}}+\left(\frac{\alpha}{\beta}\right)^{(-1)^{m+1}}-1=0\right\},
$$

where

$$
\delta_{m}=\frac{2^{m}-(-1)^{m}}{3}, \quad m \geq 0 .
$$

All curves of the form (21) accumulate at the point $Q_{\infty}(\alpha, \beta)=(1,-1)$ when $m \rightarrow \infty$. The expression (21) is easily mapped to the parameter plane $(r, \varepsilon)$ of the original map $f$ as follows

$$
H_{2^{m}}=\left\{(r, \varepsilon) \in D_{\mathcal{L M}}:(1+r)^{2 \delta_{m}}(1-\varepsilon)^{2 \delta_{m+1}}+\left(\frac{1+r}{1-\varepsilon}\right)^{(-1)^{m+1}}-1=0\right\} .
$$

Moreover, the accumulation point $Q_{\infty}$ is translated to $P_{\infty}(r, \varepsilon)=(0,2)$. In Fig. 1(a) the homoclinic bifurcation curves $H_{2^{m}}, m=0, \ldots, 4$, are numerically obtained from (23). Note that the other curves for $m>4$ also exist, but they are not observable in the presented scale, and these curves accumulate at the point $P_{\infty}$. It is also easy to check that all the homoclinic curves $H_{2^{m}}$ for any integer $m \geq 1$ intersect the line $\delta_{D F B}$. So that, depending on the value of parameter $r$ the DFB of the fixed point $x^{*}$ occurring at $\varepsilon=2$ can lead to cyclical chaotic intervals $\mathcal{Q}_{2}$ for any integer $m \geq 1$. This is not true for the curve $H_{1}$ which is asymptotic to the DFB line $\varepsilon=2$ meaning that we cannot have direct transition from a stable equilibrium point to one chaotic interval $\mathcal{Q}_{1}$. Figs. 1 (a) and (b) also display several other bifurcation curves, denoted as $\xi_{q}, q \geq 3$, plotted by dashed lines. Each such boundary corresponds to the fold BCB leading to the appearance of the two cycles $\mathcal{O}_{q}$ and $\widehat{\mathcal{O}}_{q}$ of the same period $q$. The cycle $\mathcal{O}_{q}$ is the so-called basic cycle having a single point in $I_{\mathcal{M}}$ and all other $q-1$ points in $I_{\mathcal{L}}$. The other cycle $\widehat{\mathcal{O}}_{q}$ is called complementary and it has two points in $I_{\mathcal{M}}$. For parameter values belonging to $D_{\mathcal{L M}}$ both cycles, $\mathcal{O}_{q}$ and $\widehat{\mathcal{O}}_{q}$, appear unstable. In the $(r, \varepsilon)$-parameter plane of the map $f$ the fold BCB curve at which $\mathcal{O}_{q}$ and $\widehat{\mathcal{O}}_{q}$ appear is given by

$$
\xi_{q}=\left\{(r, \varepsilon): 1-\varepsilon=-\frac{1-(1+r)^{q-1}}{r(1+r)^{q-2}}\right\}, \quad q \geq 3,
$$


then the two cycles exist in the region above the curve $\xi_{q}$. It can be proved that $\xi_{q} \cap D_{\mathcal{L M}} \neq \emptyset$ for any $q \geq 3$, and all these curves accumulate at the point $E_{\infty}(r, \varepsilon)=(1,3)$ for $q \rightarrow \infty$. As one can see in Fig. 1(a), the intersection point of each $\xi_{q}$ and the border $\delta_{B C_{\mathcal{R}}}$ is particular. In fact, as we shall see, each such point is the issuing point of other bifurcation curves located in region $D_{\mathcal{L M R}}$ and associated with cyclic chaotic intervals of the map $f$. These chaotic intervals are related to period adding structure.

3.4. All three partitions: region $D_{\mathcal{L M R}}$

The transition from region $D_{\mathcal{L M}}$ to region $D_{\mathcal{L M R}}$ occurs when $(r, \varepsilon) \in \delta_{B C_{\mathcal{R}}}$. Hence, the region $D_{\mathcal{L M R}}$ is defined as

$$
D_{\mathcal{L M R}}=\{(r, \varepsilon): 0<r<1, \varepsilon>r+2\} .
$$

For parameter values belonging to $D_{\mathcal{L M R}}$ the expression of the absorbing interval is

$$
J_{\mathcal{L M R}}=\left[f\left(x_{\mathcal{L}}\right), f\left(x_{\mathcal{R}}\right)\right]=\left[\frac{(1-r) \varepsilon}{\varepsilon-r}, \frac{(1+r) \varepsilon}{\varepsilon+r}\right]
$$

and, as it can be easily checked, $J_{\mathcal{L M R}}$ ranges across all the partitions $I_{\mathcal{L}}, I_{\mathcal{M}}$, and $I_{\mathcal{R}}$. As a consequence, the definition of the map $f$ in the region $D_{\mathcal{L M R}}$ involves all three branches. Preliminary understanding of the bifurcation structure of the region $D_{\mathcal{L M R}}$ can be obtained from Fig. 1(a). As one can see, when the parameter point moves from $D_{\mathcal{L M}}$ to $D_{\mathcal{L M R}}$ the regions associated with chaotic dynamics existing in $D_{\mathcal{L M}}$ extend as well to $D_{\mathcal{L M R}}$. In this section we describe briefly why the related boundaries change and find analytic expressions for some of them. At first, we consider the boundary $H_{1} \subset D_{\mathcal{L M}}$ related to the first homoclinic bifurcation of the fixed point $x^{*}$, and let the parameter point $p$ move along this boundary. When the parameter point crosses $\delta_{B C_{\mathcal{R}}}$ and enters the region $D_{\mathcal{L M R}}$, the condition for the first homoclinic bifurcation of the fixed point $x^{*}$ defines the new curve

$$
\widetilde{H}_{1}=\left\{(r, \varepsilon): \varepsilon=\frac{1}{r}\right\} .
$$

Similarly, all the other homoclinic bifurcation curves $H_{2^{m}} \subset D_{\mathcal{L M}}, m \geq 1$, have their extensions inside $D_{\mathcal{L M R}}$. As it can be seen in Fig. 1(a), the regions related to cyclic chaotic intervals belonging to $D_{\mathcal{L M R}}$ have more complicated structure than those belonging to $D_{\mathcal{L M}}$. Moreover, the higher the number of chaotic intervals, the higher the number of bifurcation curves bounding the related parameter regions. To explain the bifurcation structure of the region $D_{\mathcal{L M R}}$ when the parameter point crosses the curve $\widetilde{H}_{1}$, we have to consider the period adding (PA) structure of the map $f$. It is known for a generic bimodal piecewise linear map that even if its absorbing interval spreads over all three partitions the map still may have periodic orbits whose points are located only in the two outermost partitions $I_{\mathcal{L}}$ and $I_{\mathcal{R}}$. Existence conditions for such cycles can be derived by means of a discontinuous map defined in two partitions, as shown, for instance, in [9]. In particular, periodicity regions related to these cycles are arranged in the parameter space into a PA structure, known also as Arnold tongues or mode-locking tongues. However, that for the map of the form (13) the PA structure is highly degenerate and consists only of a set of lines defined by $r=$ const. To obtain the boundaries of regions constituting the PA structure (PA regions, for short) in the parameter plane of the map $f$ we use the expressions for such boundaries known for a generic bimodal piecewise linear map. Let us start with regions of the so-called first complexity level (following Leonov [5]) associated with $q$-cycles having either a single point in $I_{\mathcal{L}}$ and all other $q-1$ points in $I_{\mathcal{R}}$ (type I), or vice verse (type II). The BCB 

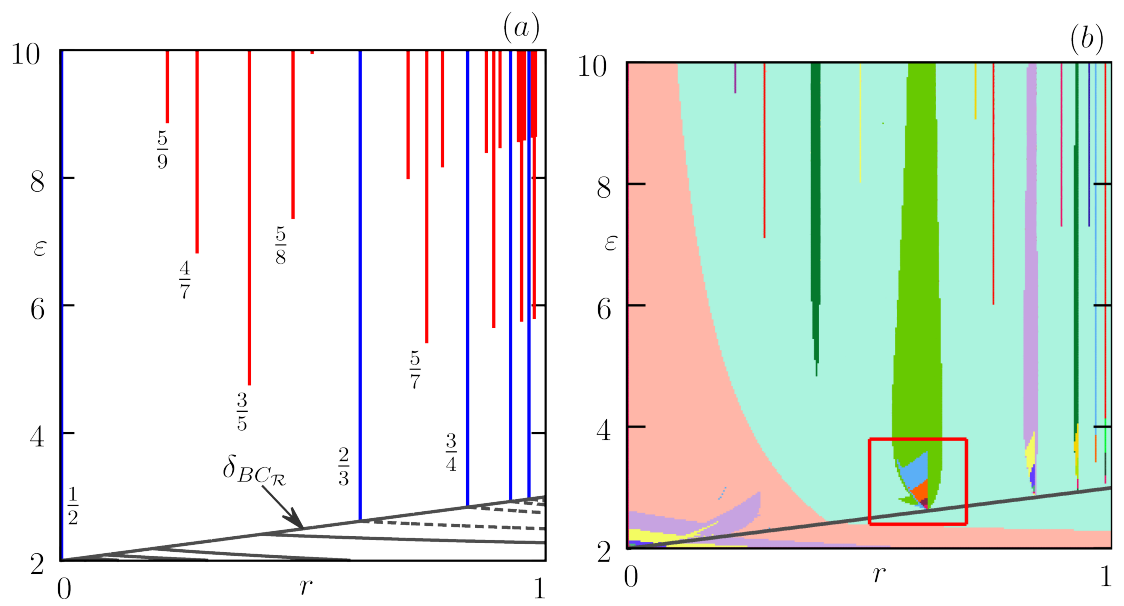

Figure 2. (a) Degenerate PA structures in the $(r, \varepsilon)$ parameter plane of the map $f$. Blue lines correspond to first complexity level cycles, red lines correspond to those of the second complexity level. (b) Numerically calculated chaoticity regions around PA structure.

boundaries of regions related to the type I cycles are defined in general form as

$$
\begin{aligned}
\text { BC with } x_{\mathcal{L}}: & \mu_{\mathcal{L}}=-\frac{1-a_{\mathcal{R}}^{q-1}}{\left(1-a_{\mathcal{R}}\right) a_{\mathcal{R}}^{q-1}} \mu_{\mathcal{R}}+\frac{1-a_{\mathcal{R}}^{q-1} a_{\mathcal{L}}}{a_{\mathcal{R}}^{q-1}} x_{\mathcal{L}}, \\
\text { BC with } x_{\mathcal{R}}: & \mu_{\mathcal{L}}=-\left(a_{\mathcal{L}}+\frac{1-a_{\mathcal{R}}^{q-2}}{\left(1-a_{\mathcal{R}}\right) a_{\mathcal{R}}^{q-2}}\right) \mu_{\mathcal{R}}+\frac{1-a_{\mathcal{R}}^{q-1} a_{\mathcal{L}}}{a_{\mathcal{R}}^{q-2}} x_{\mathcal{R}} .
\end{aligned}
$$

The BCB boundaries of the type II cycles are obtained from (27) by interchanging the indices $\mathcal{L}$ and $\mathcal{R}$ (for detail see $[1,9]$ ). Due to the fact that $\mu_{\mathcal{L}}=\mu_{\mathcal{R}}=0$ for $f$, the expressions for BCB boundaries of PA regions related to the type I cycles are clearly simplified to

$$
\text { BC with } x_{\mathcal{L}}: \frac{\left(1-a_{\mathcal{R}}^{q-1} a_{\mathcal{L}}\right) x_{\mathcal{L}}}{a_{\mathcal{R}}^{q-1}}=0, \quad \text { BC with } x_{\mathcal{R}}: \frac{\left(1-a_{\mathcal{R}}^{q-1} a_{\mathcal{L}}\right) x_{\mathcal{R}}}{a_{\mathcal{R}}^{q-2}}=0 .
$$

From (28) it is obviously deduced that for a type I cycle both BCBs, with $x_{\mathcal{L}}$ and $x_{\mathcal{R}}$, occur at the same time, namely, when $a_{\mathcal{R}}^{q-1} a_{\mathcal{L}}=1$. Similarly, for a type II cycle both BCBs occur when $a_{\mathcal{L}}^{q-1} a_{\mathcal{R}}=1$. Hence, for the map $f$ any cycle related to PA region of the first complexity level exists only when its multiplier equals to one. Taking into account that $a_{\mathcal{L}}=1+r, a_{\mathcal{R}}=1-r$ we obtain that any PA region of the first complexity level degenerates to a line $r=\bar{r}_{q, 1}$ with $\bar{r}_{q, 1}$ depending on $q$. The other regions belonging to PA structure (of the second and higher complexity levels) are degenerate as well. Having (28), we derive that for the map $f$ any PAcycle of any complexity level exists only when its multiplier equals to one, that is, for $a_{\mathcal{L}}^{l} a_{\mathcal{R}}^{q-l}=1$. Then the related PA region degenerates to a line $r=\bar{r}_{q, l}$ with $\bar{r}_{q, l}$ depending on the period of the cycle and the number of points in $I_{\mathcal{L}}$. In Fig. 2(a) we plot several lines corresponding to PA regions of the first (blue) and second (red) complexity level. Although for the map $f$ the PA structure is highly degenerate, it still plays an essential role in the bifurcation structure in the parameter plane of $f$. In particular, the set of lines composing PA structure serves as a skeleton for the regions in the parameter plane of $f$ related to the cycles of chaotic intervals shown in Fig. 2(b). In that figure the area painted light-blue corresponds to a single chaotic interval enclosing both border points, while the other colors are related to different number of chaotic intervals (as indicated in the color bar) which can enclose only one border point $\left(x_{\mathcal{L}}\right.$ 

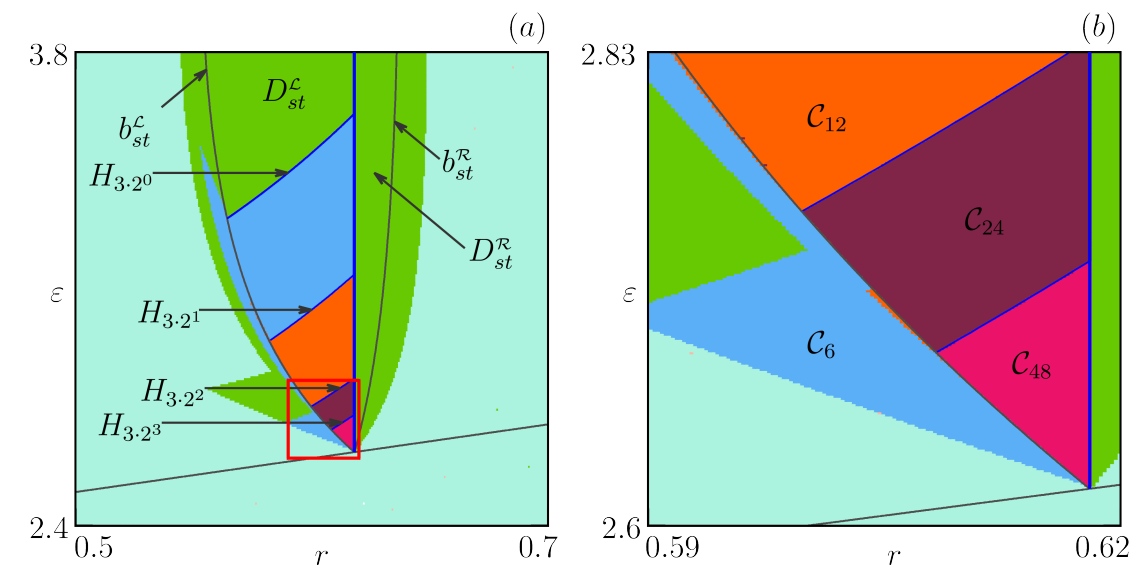

Figure 3. Chaoticity regions around the line $(1+r)^{2}(1-r)=1$ corresponding to $\mathcal{O}_{\mathcal{R L}^{2}}$. (a) In regions $D_{s t}^{\mathcal{L}}$ and $D_{s t}^{\mathcal{R}}$ the map $f^{3}$ in the neighborhood of the border point $x_{\mathcal{L}}$ is topologically conjugate to a skew tent map. Curves indicated by $H_{3 \cdot 2^{m}}$ are boundaries separating regions characterized by the existence of $3 \cdot 2^{m}$ pieces chaotic attractors. (b) Chaotic regions related to $3 \cdot 2^{m}$ pieces chaotic attractors are indicated by $\mathcal{C}_{3 \cdot 2^{m}}$.

or $x_{\mathcal{R}}$ ) or both of them. Comparing Figs. 2(a) and (b) one can see that the regions related to more than one chaotic interval are located in the neighborhood of the lines composing the PA structure. Some of the boundaries of these regions can be found analytically as described, for instance, in [10]. We concentrate below only on those boundaries which intersect with the lines composing the PA structure, and derive expressions for the region boundaries which issue from the line $r=\bar{r}_{3,2}$ associated with the cycle of period 3. In a similar way, such boundaries can be obtained for the regions related to any cycle in the PA structure. Consider $r=\bar{r}_{3,2}$, so that a cycle of period 3 having 2 points in $I_{\mathcal{L}}$ exists. First let $r<\bar{r}_{3,2}$. Following [10], we consider the map $f^{3}$ in the neighborhood of the border point $x_{\mathcal{L}}$. For the values of $r$ sufficiently close to $\bar{r}_{3,2}$ the map $f^{3}$ in the neighborhood of $x_{\mathcal{L}}$ is topologically conjugate to a skew tent map. More precisely, such a conjugacy holds until

$$
f^{3}\left(x_{\mathcal{L}}\right) \equiv f_{\mathcal{L}} \circ f_{\mathcal{R}} \circ f_{\mathcal{L}}\left(x_{\mathcal{L}}\right)<\bar{x},
$$

where $\bar{x}$ is a preimage of $x_{\mathcal{R}}$ such that $f_{\mathcal{M}}(\bar{x})=x_{\mathcal{R}}$. The equality in (29) defines a curve in the parameter plane of $f$ :

$$
\begin{aligned}
b_{s t}^{\mathcal{L}} & =\left\{(r, \varepsilon): f_{\mathcal{L}} \circ f_{\mathcal{R}} \circ f_{\mathcal{L}} \circ f_{\mathcal{M}}\left(x_{\mathcal{L}}\right)=x_{\mathcal{R}}\right\} \\
& =\left\{(r, \varepsilon): \frac{(1-\varepsilon)(1+r)^{2}(1-r)}{\varepsilon+r}+1<\frac{\varepsilon}{\varepsilon-r}\right\} .
\end{aligned}
$$

In other words, for any set of parameter values belonging to the region $D_{s t}^{\mathcal{L}}$ confined by $\mathfrak{L}_{3,2}=\left\{(r, \varepsilon): r=\bar{r}_{3,2}, \varepsilon \geq \bar{\varepsilon}_{3,2}\right\}$ and $b_{s t}^{\mathcal{L}}$, the map $f^{3}$ in the neighborhood of the border point $x_{\mathcal{L}}$ is topologically conjugate to a skew tent map $\hat{f}$ given in $(20)$ with $\alpha=(1+r)(1-r)^{2}$, $\beta=(1-r)^{2}(1-\varepsilon)$. The related boundaries of chaotic regions are then obtained from the expressions known for the skew tent map. In Fig. 3(a) we observe chaotic regions $\mathcal{C}_{3 \cdot 2^{m}}, m=0,4$. Now we turn to chaoticity regions located on the right-hand side of $\mathfrak{L}_{3,2}$, that is for $r>\bar{r}_{3,2}$. Similarly to the previous case, we consider the map $f^{3}$, but now in the neighborhood of the border point $x_{\mathcal{R}}$. The map $f^{3}$ in the neighborhood of $x_{\mathcal{R}}$ is topologically conjugate to a skew tent map $\hat{f}$ with $\alpha=(1+r)(1-r)^{2}, \beta=\left(1-r^{2}\right)(1-\varepsilon)$ as long as the inequality

$$
f^{3}\left(x_{\mathcal{R}}\right) \equiv f_{\mathcal{R}} \circ f_{\mathcal{L}} \circ f_{\mathcal{L}}\left(x_{\mathcal{R}}\right)<\hat{x},
$$


holds with $f_{\mathcal{M}} \circ f_{\mathcal{L}}(\hat{x})=x_{\mathcal{L}}$. Changing inequality to equality in (31) we get the curve

$$
\begin{aligned}
b_{s t}^{\mathcal{R}} & =\left\{(r, \varepsilon): f_{\mathcal{R}} \circ f_{\mathcal{L}} \circ f_{\mathcal{L}} \circ f_{\mathcal{M}} \circ f_{\mathcal{L}}\left(x_{\mathcal{R}}\right)=x_{\mathcal{L}}\right\}= \\
& =\left\{(r, \varepsilon): \frac{(1-\varepsilon)(1+r)^{3}(1-r)}{\varepsilon-r}+1+r<\frac{\varepsilon}{\varepsilon+r}\right\} .
\end{aligned}
$$

Then for parameter values belonging to the region $D_{s t}^{\mathcal{R}}$ confined by $\mathfrak{L}_{2,3}$ and the curve $b_{s t}^{\mathcal{R}}$ the boundaries of chaotic regions are obtained from expressions known for the skew tent map. However, as it can be observed in Fig. 3(a) for $r>\bar{r}_{3,2}$ there is only a region $\mathcal{C}_{3}$ related to three pieces chaotic attractor. It can be proved that inside $D_{s t}^{\mathcal{R}}$ no other chaotic regions appear.

\section{Concluding remarks}

In this paper we studied the map proposed in [12] to describe a cautious discrete time tâtonnement process in a pure exchange economy. The model is analytically expressed by a 1D piecewise linear bimodal map and the presence of two border points entails a complex bifurcation structure of its parameter space. Although the map has three branches it has been shown that for some parameter values its asymptotic dynamics involved only two of them. In these cases, results known for discontinuous maps or for the skew tent map have been applied. The main finding of our analyses can be resumed in the dichotomy which characterizes the dynamic behavior of the model. In fact, depending on the values of the parameters only one of the two following situations may occur: either the non trivial equilibrium point is globally stable or the orbits are chaotic. Moreover an abrupt transition characterizes the change from the first to the second regime. From an economic point of view, we may thus affirm that the introduction of the cautious process adjustment may reduce the amplitude of the price oscillations but may not eliminate the chaotic behavior typical of the discrete time tâtonnement process.

\section{Acknowledgments}

We thank the anonymous referees for their helpful comments. The work of I. Foroni and A. Panchuk has been done within the activities of the COST action IS1104 "The EU in the new economic complex geography: models, tools and policy evaluation" (www.gecomplexitycost.eu).

\section{References}

[1] Avrutin V, Schanz, M and Gardini L 2010 Calculation of bifurcation curves by map replacement Int. J. Bifurcat. Chaos 20 3105-35

[2] Day RH and Pianigiani G 1991 Statistical dynamics and economics J. Econ. Behav. Organ. 16 37-83

[3] Foroni I, Avellone A and Panchuk A 2015 Sudden transition from equilibrium stability to chaotic dynamics in a cautious tâtonnement model Preprint doi:10.1016/j.chaos.2015.05.013

[4] Ito S, Tanaka S and Nakada H 1979 On unimodal transformations and chaos II Tokyo J. Math. 2 241-59

[5] Leonov N N 1959 Map of the line onto itself Radiofisika 3 942-956

[6] Maistrenko Y L, Maistrenko V L and Chua L O 1993 Cycles of chaotic intervals in a time-delayed Chua's circuit, Int. J. Bifurcat. Chaos 3 1557-72

[7] Maistrenko Y L, Maistrenko V L, Vikul, S I and Chua L O 1995 Bifurcations of attracting cycles from time-delayed Chua's circuit Int. J. Bifurcat. Chaos 5 653-71

[8] Nusse HE and Yorke JA 1995 Border-collision bifurcation for piecewise smooth one-dimensional maps 1995 Int. J. Bifurcat. Chaos 5 189-207

[9] Panchuk A, Sushko I, Schenke B and Avrutin V 2013 Bifurcation structure in a bimodal piecewise linear map: regular dynamics Int. J. Bifurcat. Chaos 231330040.

[10] Panchuk A, Sushko I and Avrutin V 2015 Bifurcation structures in a bimodal piecewise linear map: chaotic dynamics Int. J. Bifurcat. Chaos 251530006

[11] Sushko I and Gardini L 2010 Degenerate bifurcations and border collisions in piecewise smooth 1D and 2D maps Int. J Bifurcat. Chaos 20 2045-70

[12] Weddepohl C 1995 A cautious price adjustment mechanism: chaotic behavior J. Econ. Behav. Organ. 27 293-300 